\title{
COHERENT TEMPORAL EXTRAPOLATION OF LABELED IMAGES
}

\author{
Grégoire Malandain, Gaël Michelin \\ Université Côte d'Azur, Inria, CNRS, I3S, France
}

\begin{abstract}
In developmental imaging, 3D+t series of microscopic images allow to follow the organism development at the cell level and have now became the standard way of imaging the development of living organs. Dedicated tools for cell segmentation in 3D images as well as cell lineage calculation from $3 \mathrm{D}+\mathrm{t}$ sequences have been proposed to analyze these data. For some applications, it may be desirable to interpolate label images at intermediary time-points. However, the known methods do not allow to locally handle the topological changes (ie cell. division). In the present work, we propose an extrapolation method that coherently deformed the label images to be interpolated.
\end{abstract}

Index Terms - time-lapse, cell-lineage interpolation, labeled image interpolation

\section{INTRODUCTION}

Acquiring temporal series of living data has became the standard way of imaging the motion or the evolution or the development of living organs. In developmental imaging [1], 3D+t series of microscopic images allow to follow the organism development at the cell level. Depending on the acquisition protocol and the microscopic modality, the time interval between two acquisitions may range from a few minutes (e.g. ciona embryo as in [2]), which allows a quasi realtime acquisition of the development, to several hours (e.g. arabidopsis meristem as in [3]), where several rounds of cell division may take place between two acquisitions in addition to large geometric changes.

In the later case, it may be desirable to interpolate images at intermediary time points. It may be for visualization or educational purpose (e.g. to build a movie of continuous development). This can be achieved by cross-fading from the acquisition at one acquisition time to the one at the next acquisition time (as in Fig. 3): as a consequence, all topological changes (new walls) appear simultaneously and progressively. However, to the authors' knowledge, there are no means to build an interpolated series, with grey-level or labeled images, where one can control the occurrence of cell division.

We propose in this article to design a coherent extrapolation scheme for labeled images (with known correspondences), so that segmented area border do superimpose at each intermediary timepoint. This way, we ensure a continuous deformation of the segmented areas, and we have a means to easily put any cell division at any intermediary time-point. We assume that deformations have already been computed between two successive acquisitions, and that the lineage is also known (e.g. [4]). We first recall some notions about extrapolation and interpolation on grey-level images, before describing the proposed approach. We also establish some links with nearby literature, and demonstrate the equivalence with the

Contact: \{gregoire.malandain,gael.michelin\}@inria.fr well known shape-based interpolation [5]. Implementation details are presented before the conclusion.

Experiments were conducted on two successive 3D acquisitions on a wild type plant of Arabidopsis thaliana (protocol described in [3]) whose segmentations (obtained as in [6]) have been manually corrected when necessary. Fig. 1 displays parts of homologous sections as well as their segmentations. Please note that acquisitions and processing are 3-dimensional, while presented results consist in 2D extracted sub-images.
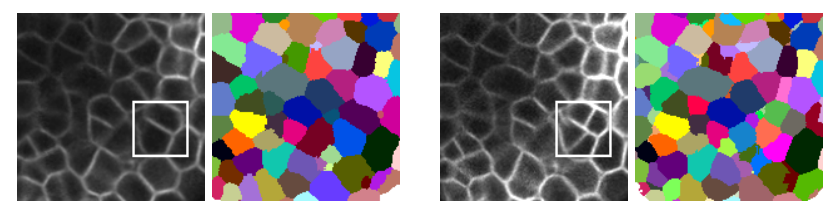

Fig. 1. Left, a sub-image extracted from a meristem volume with the associated cell segmentation. Right, a sub-image extracted from the meristem imaged 12 hours later with the associated cell segmentation. The boxes point cell divisions that occur between the acquisitions.

\section{TEMPORAL INTERPOLATION OF GREY-LEVEL IMAGES}

For the sake of simplicity, let us consider two images $I^{0}$ and $I^{1}$, and the transformation $T_{0 \leftarrow 1}$ that allows to resample $I^{0}$ into $I^{1}$ frame, i.e. to compute $I^{0} \circ T_{0 \leftarrow 1}{ }^{1}$. Estimating the image $I^{t}$ at an intermediary position $t \in] 0,1$ [ usually results from two operations. First, both $I^{0}$ and $I^{1}$ are extrapolated and resampled in the frame of $I^{t}$ and second $I^{t}$ is calculated using these two resampled images.

The first operation requires to estimate both $T_{0 \leftarrow t}$ and $T_{1 \leftarrow t}$, i.e. the transformations from $I^{t}$ towards $I^{0}$ and $I^{1}$, that enable to resample $I^{0}$ and $I^{1}$ in the frame of $I^{t}$ by $I^{0} \circ T_{0 \leftarrow t}$ and $I^{1} \circ T_{1 \leftarrow t}$. Resampling is usually achieved through bi- or tri-linear interpolation, or with more elaborate operators [7].

Let us consider a point $M_{1}$ of $I^{1}$, the transformation $T_{0 \leftarrow 1}$ pairs it with $M_{0}$ of $I^{0}$ such that $M_{0}=T_{0 \leftarrow 1} M_{1}$. Let us assume the linearity of the displacements due to the transformation: the point $M_{t}$ of $I^{t}$ paired with $M_{0}$ and $M_{1}$ through transformations $T_{0 \leftarrow t}$ and $T_{1 \leftarrow t}$ lies on the line joining $\left(M_{0}, 0\right)$ and $\left(M_{1}, 1\right)$ in the $3 \mathrm{D}+\mathrm{t}$ space (see Fig. 2(a)). $M_{t}$ can then be computed from $M_{1}$ and $T_{0 \leftarrow 1}$ :

$$
M_{t}=(1-t) M_{0}+t M_{1}=\left(t \mathbf{I d}+(1-t) T_{0 \leftarrow 1}\right) M_{1}
$$

\footnotetext{
${ }^{1}$ An integer-valued $d$-dimensional image $I$ can be defined as a function $I: \mathbb{Z}^{d} \rightarrow \mathbb{N}$. Given an interpolation method, it can be defined over $\mathbb{R}^{d}$. Geometric transformations $T$ are also functions $T: \mathbb{R}^{d} \rightarrow \mathbb{R}^{d}$, thus we will denote by $I \circ T$ the image $I$ resampled by transformation $T$.
} 


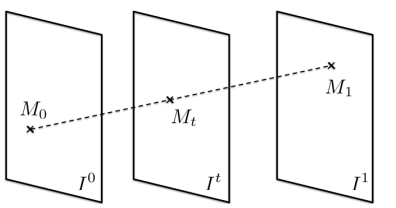

(a)

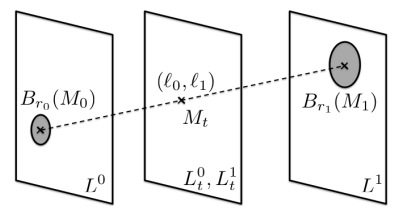

(b)
Fig. 2. Left, grey-level interpolation (sec. 2): $I^{t}\left(M_{t}\right)$ is computed from $I^{0}\left(T_{0 \leftarrow t} M_{t}\right)$ and $I^{1}\left(T_{1 \leftarrow t} M_{t}\right)$. Right, label extrapolation (sec. 3): a couple of valid labels $\left(\ell_{0}, \ell_{1}\right)$ is built at $M_{t}$ with $\ell_{0} \in B_{r_{0}}\left(T_{0 \leftarrow t} M_{t}\right)$ and $\ell_{1} \in B_{r_{1}}\left(T_{0 \leftarrow t} M_{t}\right)$.

where Id denotes the identity transformation, yielding

$$
T_{t \leftarrow 1}=t \mathbf{I d}+(1-t) T_{0 \leftarrow 1} \quad \text { and } \quad T_{1 \leftarrow t}=T_{t \leftarrow 1}^{-1}
$$

The computation of $T_{0 \leftarrow t}$ can be done by using again the assumption of linearity, which results in

$$
T_{0 \leftarrow t}=\frac{1}{1-t} \mathbf{I} \mathbf{d}-\frac{t}{1-t} T_{1 \leftarrow t}
$$

$T_{0 \leftarrow t}$ and $T_{1 \leftarrow t}$ allow to extrapolate and resample respectively $I_{0}$ and $I_{1}$ at the intermediary time $t$, for instance for a linear interpolation scheme.
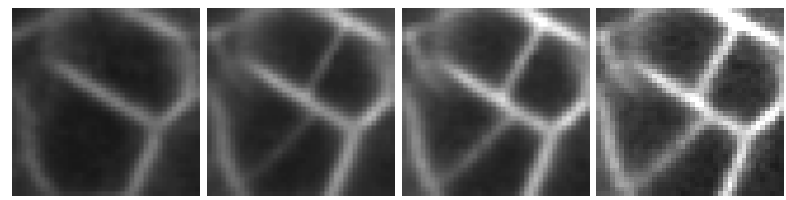

Fig. 3. The two middle images exemplified the grey-level interpolation from the left image (Fig. 1) to the right one. The inner walls indicating cell division appears progressively and simultaneously in both cells that divide, which is unrealistic.

Finally, one single image $I^{t}$ can be interpolated from the extrapolated $I^{0}$ and $I^{1}$ into $I^{t}$ frame (see Fig. 3), ie $I^{0} \circ T_{0 \leftarrow t}$ and $I^{1} \circ T_{1 \leftarrow t}$ with

$$
I^{t}=(1-t) \times I^{0} \circ T_{0 \leftarrow t}+t \times I^{1} \circ T_{1 \leftarrow t}
$$

\section{TEMPORAL EXTRAPOLATION OF LABELED IMAGES}

Let consider now label images $L^{0}$ and $L^{1}$ that correspond to the segmented grey-level images $I^{0}$ and $I^{1}$ (see Fig. 1). More precisely, $I^{0}$ (resp. $I^{1}$ ) is parted into shapes $S_{i}^{0}$ (resp. $S_{j}^{1}$ ). Values of $L^{0}$ (resp. $L^{1}$ ) are labels $\ell_{i}^{0}$ (resp. $\ell_{j}^{1}$ ) that correspond to shapes shape $S_{i}^{0}$ (resp. $\left.S_{j}^{1}\right)$.

A naive approach consists in independently extrapolating the labeled images (as a grey-level interpolation scheme will make appear intermediary values, a nearest neighbor scheme is preferred). $L^{0}$ and $L^{1}$ images can then be resampled with the transformation $T_{0 \leftarrow t}$ and $T_{1 \leftarrow t}$. This is exemplified in Fig. 4 that clearly demonstrates that shape borders do not superimpose: the blue cell of $I_{0}$ (top left image) has two children (orange and pink cells) in $I_{1}$ (bottom right image), the border of blue cell is different from the one of both orange and pink cells at any time point.

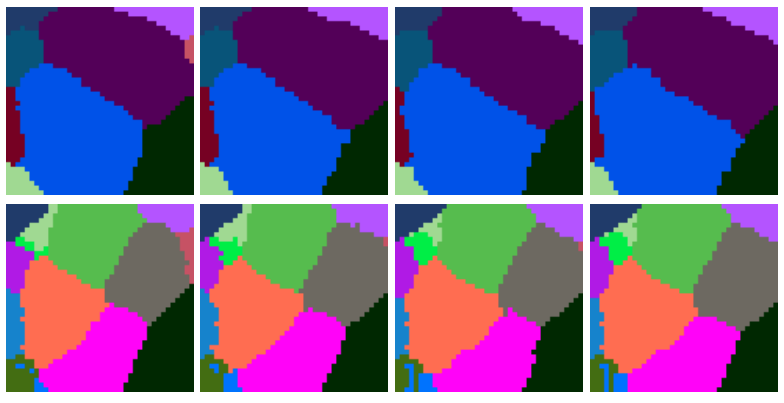

Fig. 4. Nearest neighbor resampling. First row, $L^{0}$ (at left) and its resampled versions at $t \in\{0.33,0.66,1\}$ (at right). Second row, $L^{1}$ (at right) and its resampled versions at $t \in\{0,0.33,0.66\}$.

Let also assume that lineages have been established between labels from $L^{0}$ and labels from $L^{1}$. To be more generic, let assume that a set $\mathcal{I}_{k}$ of labels from $L_{0}$ corresponds to a set $\mathcal{J}_{k}$ of labels from $L_{1}$. Typically, when studying cell lineage, $\mathcal{I}_{k}$ is composed of one shape/cell while $\mathcal{J}_{k}$ is composed of its children cells $[6,4]$. The sets $\mathcal{I}_{k}$ form a partition of $L^{0}$ (the same for $\mathcal{J}_{k}$ and $L^{1}$ ). Thus, it is desired that, in the resampled images at intermediary time points, $\bigcup_{i \in \mathcal{I}_{k}} S_{i}^{0}$ superimpose with $\bigcup_{j \in \mathcal{J}_{k}} S_{j}^{1}$.

When these correspondences are not a one-to-one mapping (i.e. when cell division arises, or when shapes appear or disappear), calculating one single interpolated image at $t$ does not make sense since it implicitly involves some choices (e.g. the time where the division occurs). Instead, we propose to extrapolate conjointly and coherently both images $L_{0}$ and $L_{1}$, that is, to build the sequences $\left\{L_{t}^{0}\right\}$ and $\left\{L_{t}^{1}\right\}$, where $L_{0}^{0}=L^{0}$ and $L_{1}^{1}=L^{1}$, and where the border of the sets $\mathcal{I}_{k}$ and $\mathcal{J}_{k}$ superimposes at every time.

A couple of labels $\left(\ell_{i}^{0}, \ell_{j}^{1}\right)$ is said to be valid if they belong to corresponding sets. Let $\mathcal{V}$ denote the set of valid couples:

$$
\left(\ell_{i}^{0}, \ell_{j}^{1}\right) \in \mathcal{V} \Leftrightarrow \exists k, i \in \mathcal{I}_{k} \text { and } j \in \mathcal{J}_{k}
$$

The set of corresponding labels for one label is denoted by

$$
\mathcal{L}\left(\ell_{i}^{0}\right)=\left\{\ell_{j}^{1} \mid\left(\ell_{i}^{0}, \ell_{j}^{1}\right) \in \mathcal{V}\right\}
$$

For $t \in] 0,1$, we want to build the label images $L_{t}^{0}$ and $L_{t}^{1}$, corresponding to the image $I^{t}$, such that the borders of corresponding sets superimpose. A point $M_{t}$ of $I^{t}$ projects respectively on $I^{0}$ and $I^{1}$ at $M_{0}$ and $M_{1}$ through the transformations $T_{0 \leftarrow t}$ and $T_{1 \leftarrow t}$, $M_{0}=T_{0 \leftarrow t} M_{t}$ and $M_{1}=T_{1 \leftarrow t} M_{t}$. The principle of the proposed method is to identify the best couple of valid labels $\left(\ell_{i}^{0}, \ell_{j}^{1}\right) \in \mathcal{V}$, with $\ell_{i}^{0}$ (resp. $\ell_{j}^{1}$ ) in the vicinity of $M_{0}$ (resp. $M_{1}$ ) as depicted by Fig. 2(b).

Let $d_{i}^{0}$ (resp. $d_{j}^{1}$ ) be the distance of $M_{0}$ (resp. $M_{1}$ ) to the shape $S_{i}^{0}$ (resp. $S_{j}^{1}$ ), this distance being obviously 0 if $M_{0} \in S_{i}^{0}$ :

$$
d_{i}^{0}=\min _{M \mid L^{0}(M)=\ell_{i}^{0}}\left\|M M_{0}\right\|
$$

The cost $c\left(\ell_{i}^{0}, \ell_{j}^{1}\right)$ of a couple of valid labels $\left(\ell_{i}^{0}, \ell_{j}^{1}\right)$ is defined by

$$
c\left(\ell_{i}^{0}, \ell_{j}^{1}\right)=(1-t) d_{i}^{0}+t d_{j}^{1}
$$

Such a formulation ensures both a continuous and smooth variation of extrapolated images with $t$ and the continuity of $L_{t}^{0}$ towards $L^{0}$ for $t \rightarrow 0$ (resp. $L_{t}^{1}$ towards $L^{1}$ for $t \rightarrow 1$ ). The best couple at 
$M_{t}$ is then chosen as the one of minimum cost, meaning that $L_{t}^{0}$ and $L_{t}^{1}$ are built by $L_{t}^{0}\left(M_{t}\right)=\ell_{\hat{\imath}}^{0}$ and $L_{t}^{1}\left(M_{t}\right)=\ell_{\hat{\jmath}}^{1}$ with $\left(\ell_{\hat{\imath}}^{0}, \ell_{\hat{\jmath}}^{1}\right)=$

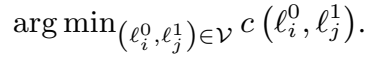

\section{RESULTS}

Fig. 5 presents the results of the proposed resampling scheme. $L_{0}^{0}=$ $L^{0}$ (upper left image) and $L_{1}^{1}=L^{1}$ (bottom right image) remain unchanged, and noticeable differences can be seen in other images when compared to Fig. 4: the border of blue cell is identical to the one of both orange and pink cells at any time point.
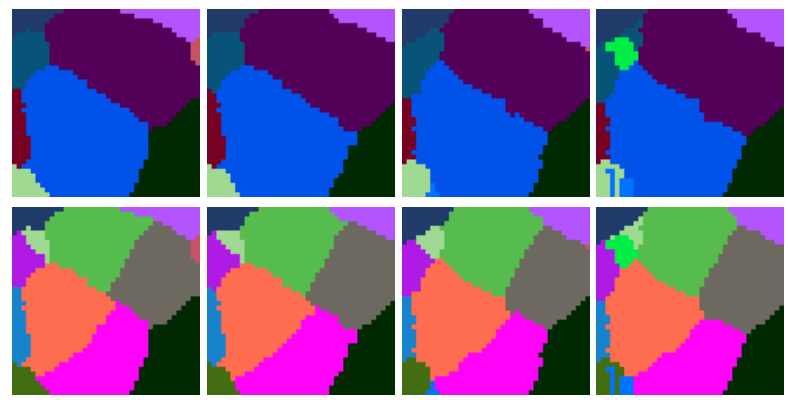

Fig. 5. First row, $L^{0}$ (at left) and its resampled versions at $t \in$ $\{0.33,0.66,1\}$ (at right). Second row, $L^{1}$ (at right) and its resampled versions at $t \in\{0,0.33,0.66\}$. Cell color are randomly chosen and similar colors may erroneously suggest topological splitting.

Having at hand border-compatible resampled label images opens the way to build simulated images. Indeed, given a couple of corresponding sets $\left(\mathcal{I}_{k}, \mathcal{J}_{k}\right)$, it is straightforward to replace the set $\mathcal{I}_{k}$ at $t$ by the set $\mathcal{J}_{k}$ at $t$. This is exemplified by Fig. 6. At left, we replace, on a voxel-to-voxel basis, the bottom cell in $L_{t}^{0}$ at $t=0.66$ by its children cells extracting from $L_{t}^{1}$ at $t=0.66$. This can be done for grey-level images too. Middle of Fig. 6 displayed the same operation, i.e. the voxel-to-voxel replacement at $t=0.66$ of the intensity values from $I_{t}^{0}$ of the set $\mathcal{I}_{k}$ by the intensity values from $I_{t}^{1}$ of the set $\mathcal{J}_{k}$ (where a new wall has appeared). Such a naive voxelto-voxel replacement is clearly not indicated for grey-level images, and a more realistic image is obtained with a progressive transition at the replaced cell border (right of Fig. 6). This last image is to be compared to the two middle images of Fig. 3.
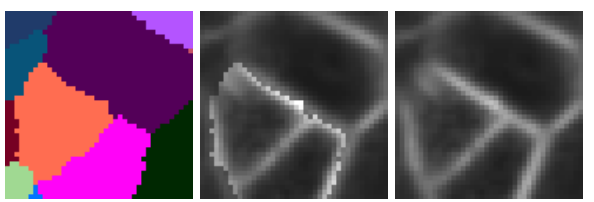

Fig. 6. Images simulated at $t=0.66$. Left, label image where only the bottom cell has divided. Middle, naive grey-level image composed of $I_{t}^{0}$ except for the dividing cell extracted from $I_{t}^{1}$. Right, same image, with a progressive transition at the replaced cell border.

\section{RELATED WORKS}

The proposed cost definition (Eq. 4) is a weighted sum of positive (or null) distances, which looks similar to the one used in shapebased interpolation [5] (SBI) where distances may also be negative. We demonstrate that the proposed approach is equivalent to SBI, according transformations are equal to identity and label images are binary. Indeed, in shape-based interpolation, a distance map is computed from the shape interface, with a (say) negative distance inside the shape $S$ and positive outside. The iso-surface at 0 defines then the object. From the distance maps $D^{0}$ and $D^{1}$ issued from $I^{0}$ and $I^{1}$, an image $D^{t}$ is computed to characterize the shape $S_{t}$ at $t$

$$
D^{t}(M)=(1-t) D^{0}(M)+t D^{1}(M)
$$

The binary shape at $t$ is deduced from the negative values of $D^{t}$. The interpolation occurs when $D^{0}(M)$ and $D^{1}(M)$ have opposite signs. Let us consider $D^{0}(M)<0\left(M\right.$ is inside $\left.S^{0}\right)$ and $D^{1}(M)>0(M$ is outside $S^{1}$ ) without loss of generality. We have

$$
\begin{aligned}
M \in S_{t} & \Leftrightarrow(1-t) D^{0}(M)+t D^{1}(M)<0 \\
\text { and } M \notin S_{t} & \Leftrightarrow t D^{1}(M)<-(1-t) D^{0}(M) \\
& \Leftrightarrow t D^{1}(M)>-(1-t) D^{0}(M)
\end{aligned}
$$

Say now that these images are two-labeled ( $\ell$ for the inside and $\bar{\ell}$ for the outside). $M$ being inside $S_{0}$, we have $d_{\ell}^{0}=0$ (zero distance to the inside) and $d_{\bar{\ell}}^{0}=-D^{0}(M)$ (recall that $D^{0}(M)<0$ ). $M$ being outside $S_{1}$, we have $d_{\bar{\ell}}^{1}=0$ and $d_{\ell}^{1}=D^{1}(M)$. With the proposed approach, to decide whether $M$ at $t$ should be labeled either $\ell$ (inside) or $\bar{\ell}$ (outside) comes to compare the costs $(1-t) d_{\ell}^{0}+t d_{\ell}^{1}$ and $(1-t) d_{\bar{\ell}}^{0}+t d_{\bar{\ell}}^{1}$ and to choose the label giving the minimum

$$
\begin{aligned}
M \in S_{t} & \Leftrightarrow(1-t) d_{\ell}^{0}+t d_{\ell}^{1}<(1-t) d_{\bar{\ell}}^{0}+t d_{\bar{\ell}}^{1} \\
& \Leftrightarrow t d_{\ell}^{1}<(1-t) d_{\bar{\ell}}^{0} \\
& \Leftrightarrow t D^{1}(M)<-(1-t) D^{0}(M)
\end{aligned}
$$

Inequality (6) is identical to condition (5), which demonstrate the equivalence.

SBI have also been used in a multi-label context, e.g. to build an average atlas from a set of labeled images in [8]. Their method was designed for one-to-one correspondences, and not for many-to-many correspondences as enabled here. Moreover, the proposed (naive) implementation is less efficient than the one proposed in this article. Last, it only builds the average label image, and not a weighted average as proposed here.

\section{IMPLEMENTATION DETAILS}

A naive implementation may consists in computing distances in $L^{0}$ and $L^{1}$ for each pair of corresponding sets, and computing the best couple from these distance maps (as described in [8]). This is clearly inefficient since for most of the points $M_{t}$ (more than $80 \%$ of the voxels in our experiments), the labels associated to its projections into $I^{0}$ and $I^{1}\left(M_{0}=T_{0 \leftarrow t} M_{t}\right.$ and $\left.M_{1}=T_{1 \leftarrow t} M_{t}\right)$ are a valid couple of labels (see Fig. 7): the associated distance for both $M_{0}$ and $M_{1}$ is 0 , so is the cost, and this is an absolute minimum.

Thus, we propose to search for labels into spheres $B_{r_{0}}\left(M_{0}\right)$ and $B_{r_{1}}\left(M_{1}\right)$ of increasing radii $r_{0}$ and $r_{1}$ centered at $M_{0}$ and $M_{1}$. We start with $r_{0}=r_{1}=0$ (i.e. with the couple $\left(L^{0}\left(M_{0}\right), L^{1}\left(M_{1}\right)\right)$ ) and let increase $r_{0}$ and $r_{1}$ by $\delta r_{0}$ and $\delta r_{1}$ until a valid couple $\left(\ell_{i}^{0}, \ell_{j}^{1}\right) \in \mathcal{V}$ is found. To increase the radii at most by 1 , we chose

$$
\left\{\begin{array}{lll}
t<0.5 & \delta r_{0}=\frac{t}{1-t} & \delta r_{1}=1 \\
t>0.5 & \delta r_{0}=1 & \delta r_{1}=\frac{1-t}{t}
\end{array}\right.
$$




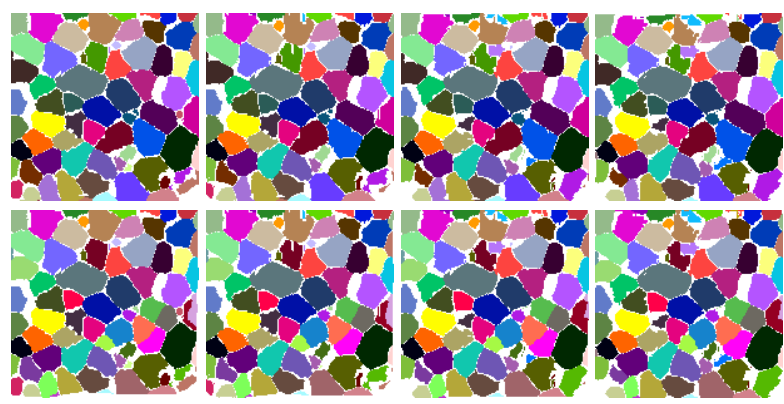

Fig. 7. First row, $L^{0}$ (at left) and its resampled versions at $t \in$ $\{0.33,0.66,1\}$ (at right). Second row, $L^{1}$ (at right) and its resampled versions at $t \in\{0,0.33,0.66\}$. Color points out voxels where nearest neighbor interpolation yields corresponding sets; white areas (at cell border) indicates that corresponding sets have to be searched further.

Let us assume we found $\left(\ell_{i}^{0}, \ell_{j}^{1}\right) \in \mathcal{V}$ with $\ell_{i}^{0} \in L^{0} \cap B_{r_{0}}\left(M_{0}\right)$ and $\ell_{j}^{1} \in L^{1} \cap B_{r_{1}}\left(M_{1}\right)$. We have to check whether a "better" couple $\left(\ell_{i^{\prime}}^{0}, \ell_{j^{\prime}}^{1}\right)$ can be found if we still increase $r_{0}$ and $r_{1}$, verifying

$$
(1-t) d_{i^{\prime}}^{0}+t d_{j^{\prime}}^{1}=c\left(\ell_{i^{\prime}}^{0}, \ell_{j^{\prime}}^{1}\right)<c\left(\ell_{i}^{0}, \ell_{j}^{1}\right)=(1-t) d_{i}^{0}+t d_{j}^{1}
$$

For such a couple $\left(\ell_{i^{\prime}}^{0}, \ell_{j^{\prime}}^{1}\right)$ we have either $\ell_{i^{\prime}}^{0} \notin L^{0} \cap B_{r_{0}}\left(M_{0}\right)$ or $\ell_{j^{\prime}}^{1} \notin L^{1} \cap B_{r_{1}}\left(M_{1}\right)$ (otherwise $\left(\ell_{i^{\prime}}^{0}, \ell_{j^{\prime}}^{1}\right)$ would already have been compared to $\left.\left(\ell_{i}^{0}, \ell_{j}^{1}\right)\right)$.

Without loss of generality, let us assume $\ell_{i^{\prime}}^{0} \notin L^{0} \cap B_{r_{0}}\left(M_{0}\right)$. It comes that $d_{i^{\prime}}^{0}>r_{0} \geq d_{i}^{0}$ and for Eq. (6) to stand, we need $d_{j^{\prime}}^{1}<d_{j}^{1}$. We have also $\ell_{i^{\prime}}^{0} \in \mathcal{L}\left(\ell_{j^{\prime}}^{1}\right)$. Thus a necessary condition for $\left(\ell_{i^{\prime}}^{0}, \ell_{j^{\prime}}^{1}\right)$ to exist is $\exists \ell_{j^{\prime}}^{1}, \ell_{i^{\prime}}^{0}$ such that

$$
\begin{aligned}
& \text { either } \begin{cases} & \ell_{j^{\prime}}^{1} \in L^{1} \cap B_{r_{1}}\left(M_{1}\right) \mid d_{j^{\prime}}^{1}<d_{j}^{1} \\
\text { and } \quad \ell_{i^{\prime}}^{0} \in \mathcal{L}\left(\ell_{j^{\prime}}^{1}\right) \text { with } \ell_{i^{\prime}}^{0} \notin L^{0} \cap B_{r_{0}}\left(M_{0}\right)\end{cases} \\
& \quad \text { or } \begin{cases}\ell_{i^{\prime}}^{0} \in L^{0} \cap B_{r_{0}}\left(M_{0}\right) \mid d_{i^{\prime}}^{0}<d_{i}^{0} \\
\text { and } \quad \ell_{j^{\prime}}^{1} \in \mathcal{L}\left(\ell_{i^{\prime}}^{0}\right) \text { with } \ell_{j^{\prime}}^{1} \notin L^{1} \cap B_{r_{1}}\left(M_{1}\right)\end{cases}
\end{aligned}
$$

Let us define the set $J^{\prime}$ (and similarly $I^{\prime}$ ) of labels $\ell_{j^{\prime}}^{1}$ in $L^{1} \cap$ $B_{r_{1}}\left(M_{1}\right)$ that have corresponding labels not in $L^{0} \cap B_{r_{0}}\left(M_{0}\right)$, i.e.

$$
\begin{aligned}
J^{\prime}= & \left\{\ell_{j^{\prime}}^{1} \in L^{1} \cap B_{r_{1}}\left(M_{1}\right) \mid d_{j^{\prime}}^{1}<d_{j}^{1}\right. \text { and } \\
& \left.\exists \ell_{i^{\prime}}^{0} \in \mathcal{L}\left(\ell_{j^{\prime}}^{1}\right) \text { with } \ell_{i^{\prime}}^{0} \notin L^{0} \cap B_{r_{0}}\left(M_{0}\right)\right\}
\end{aligned}
$$

If both sets $J^{\prime}$ and $I^{\prime}$ are empty, then $\left(\ell_{i}^{0}, \ell_{j}^{1}\right)$ is the global minimum. In our experiment, this condition was always sufficient to stop the search. For completeness, let study the case where one of the set is non-empty, say $J^{\prime} \neq \emptyset$. We exhibit then a bound on the radius $r_{0}$ for the search of $\ell_{i^{\prime}}^{0}$.

Let $d_{J^{\prime}}^{1}=\min _{j^{\prime} \in J^{\prime}} d_{j^{\prime}}^{1} . \ell_{i^{\prime}}^{0}$ is to be searched in $L^{0} \cap B_{R_{0}}\left(M_{0}\right)$ with $R_{0}>r_{0}$. A bound on $R_{0}$ can be computed since we want Eq. (6) to be verified.

$$
\begin{aligned}
& (1-t) R_{0}+t d_{J^{\prime}}^{1} \leq(1-t) R_{0}+t d_{j^{\prime}}^{1}<c\left(\ell_{i}^{0}, \ell_{j}^{1}\right) \\
\Leftrightarrow \quad & R_{0}<\frac{1}{1-t}\left(c\left(\ell_{i}^{0}, \ell_{j}^{1}\right)-t d_{J^{\prime}}^{1}\right)=d_{i}^{0}+\frac{t}{1-t}\left(d_{j}^{1}-d_{J^{\prime}}^{1}\right)
\end{aligned}
$$

Equivalently, if $I^{\prime} \neq \emptyset$, the bound $R_{1}$ on $r_{1}$ is

$$
R_{1}<d_{j}^{1}+\frac{1-t}{t}\left(d_{i}^{0}-d_{I^{\prime}}^{0}\right)
$$

Hence, once we found a valid couple $\left(\ell_{i}^{0}, \ell_{j}^{1}\right) \in \mathcal{V}$, we check whether the condition (7) is verified. If no, we can stop. If yes, we compute the bounds $R_{0}$ and/or $R_{1}$ and keep $r_{0}$ and/or $r_{1}$ increasing until either the condition (7) is no more verified or the bounds are reached.

\section{CONCLUSION}

We proposed in this article a method that extrapolates two series of coherent segmentations between two given segmentations and both the transformation and the many-to-many mapping that relies them. We demonstrated the equivalence with the shape-based interpolation method, and proposed an efficient implementation method. Such a method allows to interpolate temporally continuous (as cell borders or union of cell borders) from one image to the next as well as introducing cell division at any desired time point. While used in connection with biological knowledge, this allows to build realistic movies of organism development. In addition, such an approach may be easily extrapolated to patient-dedicated atlas construction, by a weighted sum of known atlases. The proposed method can easily be extended to other weighting functions than distances (e.g. log odds [9]), although it should not change drastically the results in our targeted applications.

Acknowledgment: Authors are funded by the following grants: IPL Morphogenetics and ANR Dig-Em. Data are courtesy of Y. Refahi, R. Wightman and H. Jönsson (Sainsbury Lab., Cambridge univ.).

\section{REFERENCES}

[1] P.J. Keller, "Imaging morphogenesis: Technological advances and biological insights," Science, vol. 340, no. 6137, pp. 1234168+, June 2013.

[2] L. Guignard, C. Godin, U.M. Fiuza, L. Hufnagel, P. Lemaire, and G. Malandain, "Spatio-temporal registration of embryo images," in ISBI, Pekin, China, Apr. 2014, IEEE.

[3] G. Michelin, Y. Refahi, R. Wightman, H. Jönsson, J. Traas, C. Godin, and G. Malandain, "Spatio-temporal registration of 3D microscopy image sequences of Arabidopsis floral meristems," in ISBI, Prague, Czech Republic, Apr. 2016.

[4] F. Amat, B. Hockendorf, Y. Wan, W.C. Lemon, K. McDole, and P.J. Keller, "Efficient processing and analysis of large-scale light-sheet microscopy data," Nat Protoc, vol. 10, no. 11, pp. 1679-96, November 2015.

[5] S.P. Raya and J.K. Udupa, "Shape-based interpolation of multidimensional objects," IEEE Trans Med Imaging, vol. 9, no. 1, pp. 32-42, 1990.

[6] R. Fernandez, P. Das, V. Mirabet, E. Moscardi, J. Traas, J.L. Verdeil, G. Malandain, and C. Godin, "Imaging plant growth in 4-d: robust tissue reconstruction and lineaging at cell resolution," Nat Meth, vol. 7, pp. 547-553, 2010.

[7] P. Thévenaz, T. Blu, and M. Unser, "Interpolation revisited," IEEE Transactions on Medical Imaging, vol. 19, no. 7, pp. 739758, July 2000.

[8] T. Rohlfing and C.R. Maurer, "Shape-based averaging," IEEE Trans Image Process, vol. 16, no. 1, pp. 153-61, January 2007.

[9] K.M. Pohl, J. Fisher, S. Bouix, M.E. Shenton, R.W. McCarley, W.E.L. Grimson, R. Kikinis, and W.M. Wells, "Using the logarithm of odds to define a vector space on probabilistic atlases," Medical Image Analysis, vol. 11, no. 6, pp. 465-477, 2007. 